\title{
Microstructure of MC-Fe Composite Layer on Carbon Steel by Laser Surface Alloying
}

\author{
Takuto YAMAGUCHI ${ }^{* 1,2}$, Hideki HAGINO ${ }^{{ }_{1}}$, Mamoru TAKEMURA ${ }^{* 1}$ and Atsushi NAKAHIRA ${ }^{* 2}$ \\ ${ }^{* 1}$ Technology Research Institute of Osaka Prefecture,2-7-1, Ayumino, Izumi, Osaka 594-1157, Japan \\ ${ }^{*}$ Osaka Prefecture University, 1-1 Gakuen-cho, Sakai, Osaka 599-8531, Japan \\ Email address: t_yamaguchi@tri-osaka.jp
}

\begin{abstract}
Laser alloying using carbide forming elements ( $\mathrm{Ti}, \mathrm{Nb}$, and $\mathrm{V}$ ) was carried out to form an $\mathrm{MC}$ Fe composite coating on a carbon steel substrate for improving wear resistance. The microstructure of laser alloyed zone was studied by OM, SEM/EDS, STEM, and XRD to reveal the effect of additional elements and laser alloying conditions on the distribution of carbides. The laser alloyed zone was found to consist of MC carbide and iron matrix. Hardness and wear properties of the laser alloyed zone were improved as compared with the laser transformation hardened sample. Dispersion of carbide within the laser alloyed zone is shown to be effective in decreasing the friction coefficient and improving wear resistance.
\end{abstract}

DOI: $10.2961 / \mathrm{jlmn} .2014 .02 .0001$

Keywords: laser surface alloying, microstructure, metal matrix composite, carbide, steel

\section{Introduction}

Metal matrix composites (MMC) that use ceramic particles for reinforcement have good toughness and wear resistance when applied to wear resistant materials such as tools and die [1]. MMCs are generally produced by a powder metallurgy or casting processes. In the powder metallurgy process, it is difficult to produce large and complex shaped components. In contrast, complex shapes can be produced using the casting process, however, it requires a lot of energy to melt a high melting temperature matrix such as iron. However, it is efficient if MMC coatings are locally formed on only the required area of low cost materials by using a laser surface modification process. Recently, a number of researchers have investigated MMC coating using laser cladding or laser surface alloying [2-6].

Laser surface alloying is one route of producing an MMC coating on a substrate. This process consists of melting the surface of a substrate with additional materials, mixing these components, followed by rapid solidification of the mixture [7-9]. Advantages of this process are as follows: low thermal strain, fine microstructure, and flexibility of the choice of substrates and additional materials. MMC coatings can be produced by injection of hard particles into the molten matrix [10-11] or in-situ synthesis of hard particles in the molten pool [12-13].

In this study, laser surface alloying using graphite and pure carbide forming elements such as titanium, niobium, and vanadium was carried out to form MC-Fe composite coating on carbon steel in order to improve wear resistance. Carbon steel is a typical low cost industrial material, and $\mathrm{MC}$ carbides such as $\mathrm{TiC}, \mathrm{NbC}, \mathrm{VC}$, have high hardness and are chemically stable in a ferrous matrix, hence each carbide is effective for improving wear resistance [14].

The issue of MMC coating by the laser surface alloying process is to control the distribution of reinforcements and to enhance the uniformity of the coating layer. In the laser alloying process, melting and solidification occur in a very short time. The mechanism of the formation of microstructures in this rapid process is not well understood. Size, morphology, and volume fraction of reinforcements have significant effects on the mechanical properties, furthermore, it is difficult to control the parameters of these reinforcements. We have already reported about $\mathrm{TiC}$ reinforced ferrous coating by laser surface alloying using graphite and pure titanium powder, and revealed the relationship between $\mathrm{TiC}$ distribution and process parameters [15].

In this study, we adopt the same procedure applied in other carbide systems and investigate the difference in microstructure of the laser alloyed zone. The purpose of this paper is to reveal the effect of various kinds of additional elements on the carbide dispersion state and wear property.

\section{Experimental procedure}

A medium carbon steel (JIS:S50C) was used as a substrate. Table 1 shows the chemical composition of the substrate. Pure titanium, niobium, and vanadium powder were used as additional materials. These powders were mixed with pure graphite powder at the weight ratio of $\mathrm{C}: \mathrm{Ti}=1: 4$, $\mathrm{C}: \mathrm{Nb}=1: 8$, and $\mathrm{C}: \mathrm{V}=1: 4$. These powder mixtures were diluted with ethyl alcohol to obtain a pasty mixture. The paste was applied onto the surface of the substrate. The thickness of the pre-coated paste was $0.2 \mathrm{~mm}$.

The laser used in this study is a fiber-coupled diode laser (Laserline : LDL-160-1000) of which the wavelength is a mixture of $808 \mathrm{~nm}$ and $940 \mathrm{~nm}$, and the maximum output power is $1 \mathrm{~kW}$. The laser beam was transmitted through an optical fiber of $1 \mathrm{~mm}$ core diameter, and focused on the specimen surface by a lens of $100 \mathrm{~mm}$ focal

Table 1 Chemical composition of substrate. (mass\%) 


\begin{tabular}{cccccc}
\hline $\mathrm{C}$ & $\mathrm{Si}$ & $\mathrm{Mn}$ & $\mathrm{P}$ & $\mathrm{S}$ & $\mathrm{Fe}$ \\
\hline 0.51 & 0.22 & 0.66 & 0.014 & 0.006 & Bal. \\
\hline
\end{tabular}

Table 2 Laser alloying conditions.

\begin{tabular}{ll}
\hline Laser & Diode laser \\
Wavelength & $808 \mathrm{~nm}$ and $940 \mathrm{~nm}$ \\
Spot diameter & $1 \mathrm{~mm}$ \\
Traverse speed & $2,5,10 \mathrm{~mm} / \mathrm{s}$ \\
$\begin{array}{l}\text { Thickness of pre-coated } \\
\text { powder mixture }\end{array}$ & $0.2 \mathrm{~mm}$ \\
Shield gas & $\operatorname{Ar}(201 / \mathrm{min})$ \\
\hline
\end{tabular}

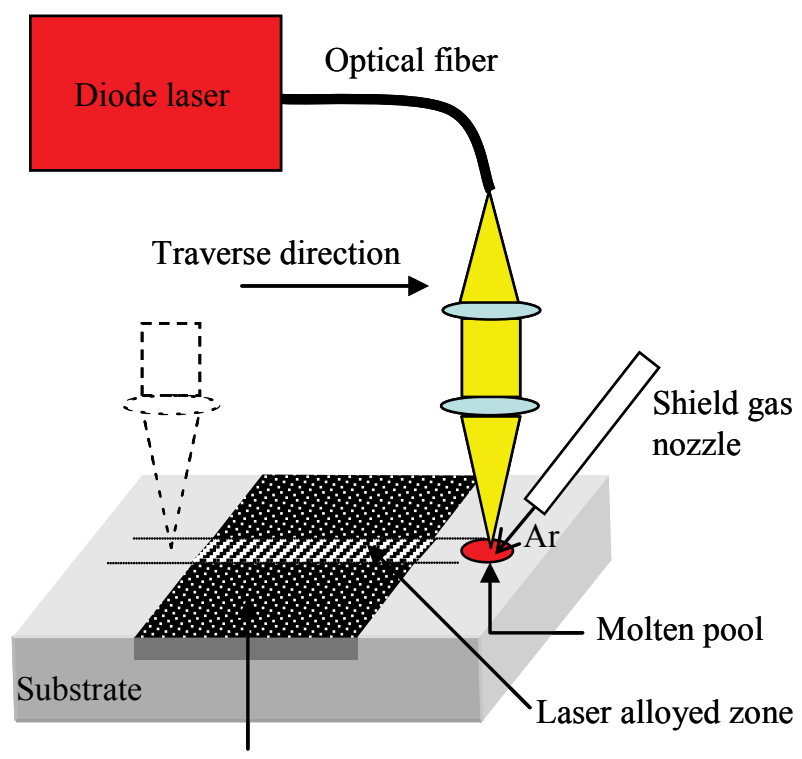

Pre-coated powder mixture of ( $\mathrm{M}+$ graphite $)$

Fig.1 Schematic illustration of experimental setup.

length. The spot diameter on the specimen surface was 1 $\mathrm{mm}$. The specimens were traversed relative to the laser beam, using a numerically controlled $\mathrm{X}-\mathrm{Y}$ table. The traverse speed was varied within the range of 2 to $10 \mathrm{~mm} / \mathrm{s}$, and argon was used as the shielding gas. Table 2 shows the laser alloying conditions and Fig.1 shows the experimental setup of the laser alloying.

The microstructure and the composition of the laser alloyed zone were studied using optical microscopy (OM), SEM/EDS. X-Ray diffraction using $\mathrm{Cu} \mathrm{K} \alpha$ radiation was performed for phase identification. The details of carbide particles and substructure of the matrix were studied using scanning transmission electron microscopy (STEM, Hitachi HD2700). The samples for STEM observation were prepared by FIB (Hitachi FB2200).

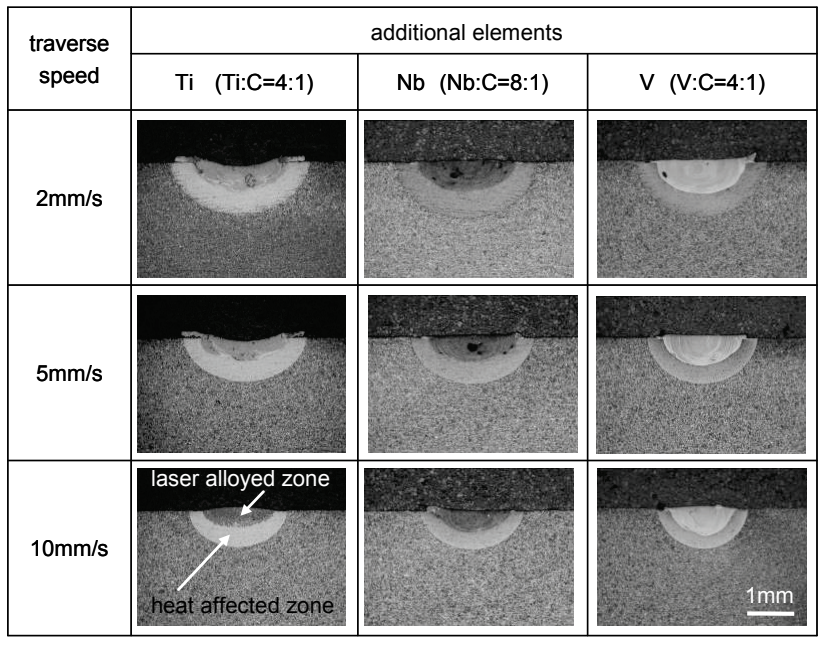

Fig.2 Cross sections of samples.

Vickers micro-hardness measurements, using a load of $2.94 \mathrm{~N}$, were carried out on a polished cross-section. Wear testing using a reciprocating wear tester was carried out, and profiles of the wear scars were measured using a contour form measuring instrument to evaluate the amount of wear after wear testing.

\section{Results and discussion}

\subsection{Macrographs of cross section}

Fig. 2 shows the cross section of samples after laser alloying treatment. The laser alloyed zone contains a few voids, however no cracks were observed. The width of the laser alloyed zone was about $1.5 \mathrm{~mm} \sim 2.0 \mathrm{~mm}$ slightly larger than the laser beam diameter. The depth at the center of laser alloyed zone was about $0.4 \mathrm{~mm} \sim 0.6 \mathrm{~mm}$. The area of the laser alloyed zone becomes smaller at a higher traverse speed because the heat input decreases with increasing the traverse speed. The difference from additional elements had little effect on the size of laser alloyed zone.

\subsection{Microstructure}

Fig.3 (a) shows SEM image of the substrate before laser irradiation. In this image, ferrite and pearlite (lamellar structure of ferrite and $\mathrm{Fe}_{3} \mathrm{C}$ ) microstructure was observed.

Fig.3 (b) (d) shows BSE compositional images of the laser alloyed zone. From the results of EDS analysis, each laser alloyed zone contains, $6.2 \mathrm{wt} \%$ Ti(Fig.3(b)), $9.7 \mathrm{wt} \%$ $\mathrm{Nb}$ (Fig.3(c)), and $6.7 \mathrm{wt} \% \mathrm{~V}$ (Fig.3(d)).

In the laser alloyed zone of the Ti-alloyed and $\mathrm{Nb}$ alloyed samples, discrete second phase particles (about 1-2 $\mu \mathrm{m})$ were observed. These particles exhibit a dendritic, flower-like, and near-equiaxed shape. Rod-shaped particles (less than $1 \mu \mathrm{m}$ ) and a lamellar structure were also observed in the inter-cellular region of the matrix. In the Valloyed sample, discrete particles were not observed, and only a lamellar structure was observed in the inter-dendrite region of the matrix.

Fig.4 shows XRD results of the surface of the substrate and each laser alloyed zone. Contrary to the SEM image (Fig.3(a)), the peak of $\mathrm{Fe}_{3} \mathrm{C}$ was unclear in the spectrum 

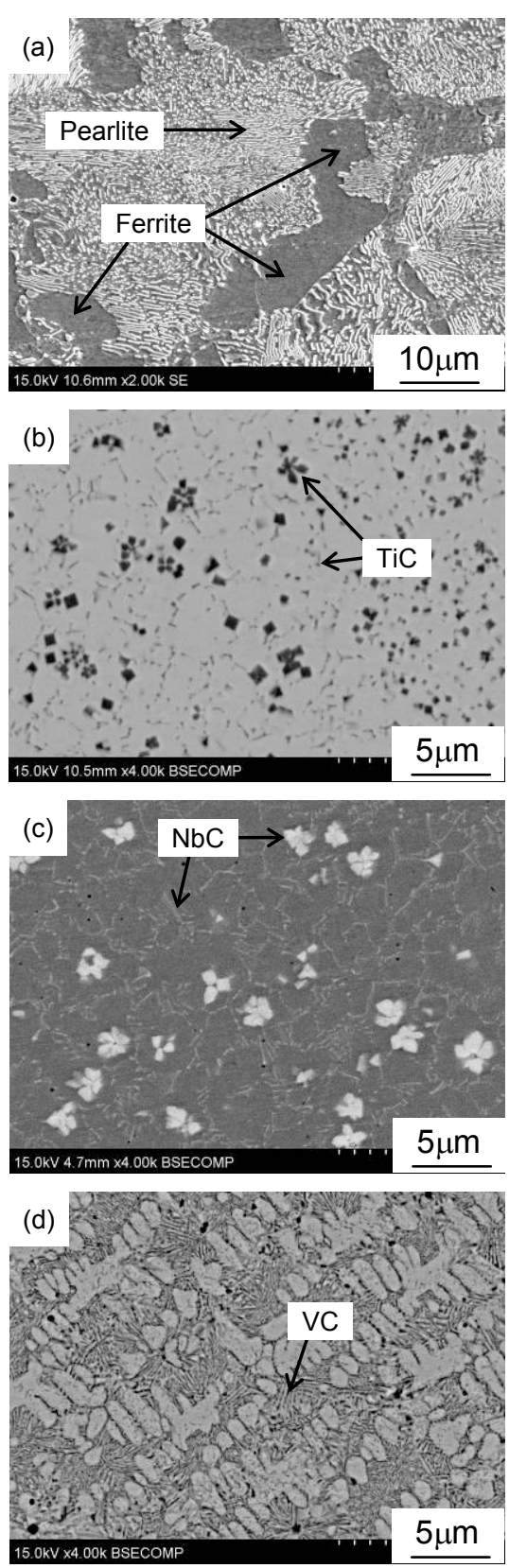

Fig.3 (a) SE image of substrate (non-laser irradiated zone) (b) (d) BSE Compositional images of laser alloyed zone. (b) Ti alloyed zone, (c)Nb alloyed zone, (d)V alloyed zone (Power 720W, Traverse speed $5 \mathrm{~mm} / \mathrm{s}$ )

of the substrate (Fig.4(d)). The reason is that the peak intensity of $\mathrm{Fe}_{3} \mathrm{C}$ is relatively low in contrast to that of the bcc-iron matrix.

In the laser alloyed zone of Ti-alloyed and $\mathrm{Nb}$-alloyed samples, peaks of bcc-iron and MC carbides such as $\mathrm{TiC}$ and $\mathrm{NbC}$ were clearly observed.

In contrast, the peak of $\mathrm{VC}$ carbide was not clear in the V-alloyed sample, but the peak of fcc-iron was observed. From the results of elemental analysis by EDS and EPMA, each additional elements ( $\mathrm{Ti}, \mathrm{Nb}$, and $\mathrm{V}$ ) and carbon were simultaneously detected at the area of second phase of Fig.3(b) (d). According to these results, it seems that the second phase particles in Fig. 3 were MC carbides.

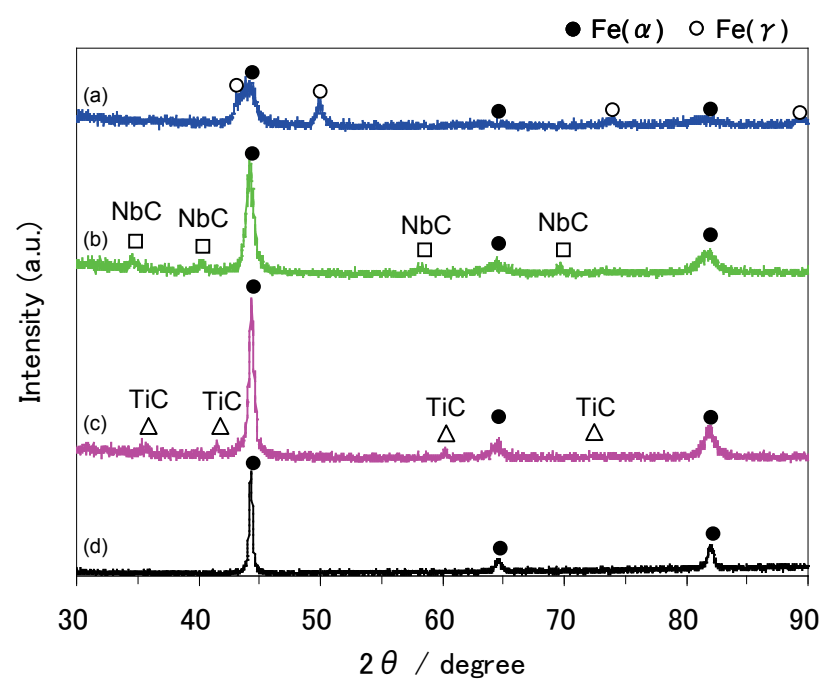

Fig. 4 XRD results of samples.

(a)V alloyed zone, (b)Nb alloyed zone, (c)Ti alloyed zone (Power 720W, Traverse speed $5 \mathrm{~mm} / \mathrm{s}$ )

(d) Non-laser irradiated substrate.
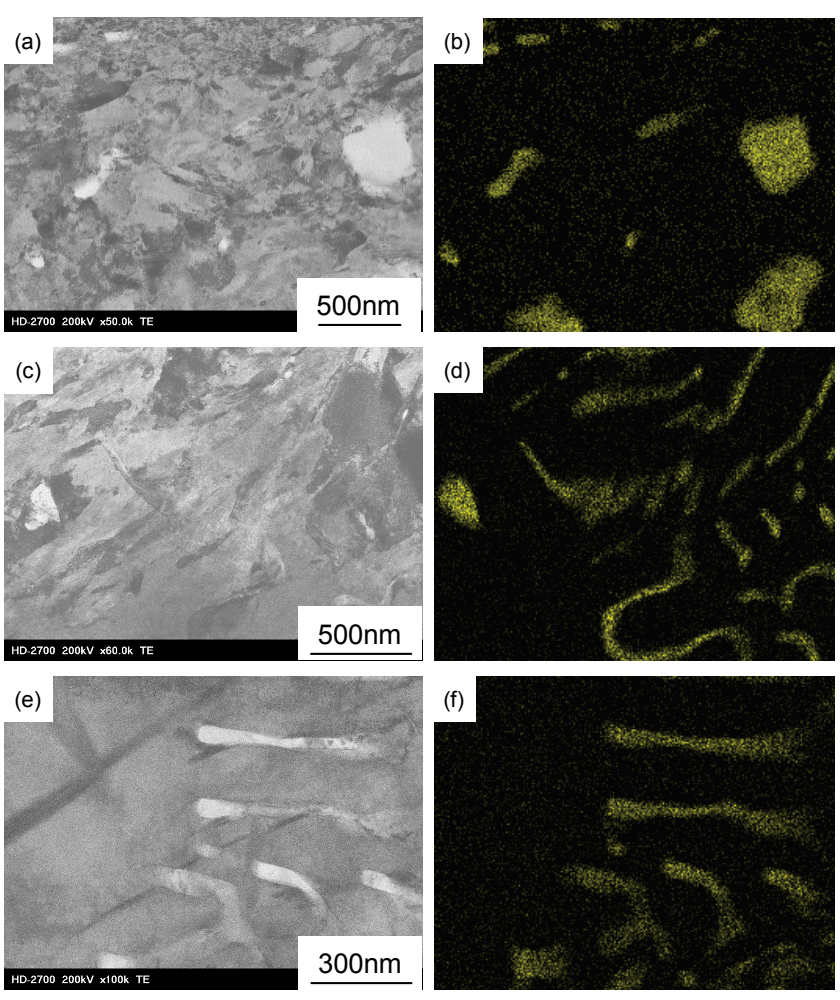

Fig.5 Bright field (BF) images and elements distribution by STEM/EDS.
(a)BF image and (b)Ti distribution of Ti alloyed zone
(c)BF image and (d) $\mathrm{Nb}$ distribution of $\mathrm{Nb}$ alloyed zone
(e)BF image and (f) $\mathrm{V}$ distribution of $\mathrm{V}$ alloyed zone

It seems that the discrete particles of $\mathrm{TiC}$ and $\mathrm{NbC}$ (Fig.3 (a), (b)) are primary carbides precipitated from $\mathrm{Fe}$ M-C liquid, and fine rod-shaped particles and lamellar structures are formed by eutectic solidification of carbide and iron matrix.

Fig.5 shows STEM bright-field images and the elements distribution of the laser alloyed zone. In these images, the $\mathrm{MC}$ carbides precipitated in the matrix were observed. In 


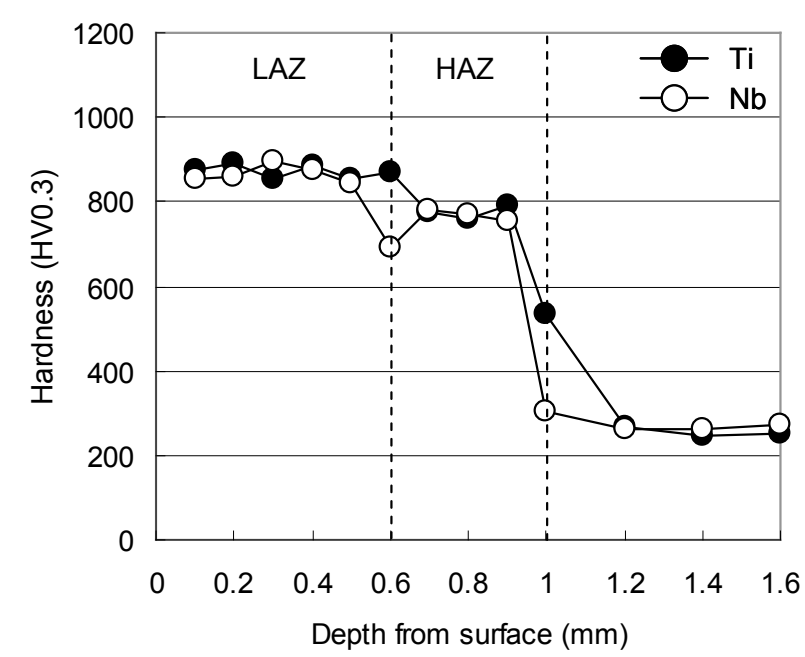

Fig.6 Hardness depth profiles of Ti alloyed sample and $\mathrm{Nb}$ alloyed sample. (Traverse speed $5 \mathrm{~mm} / \mathrm{s}$ ) (LAZ:laser alloyed zone, HAZ:heat affected zone)

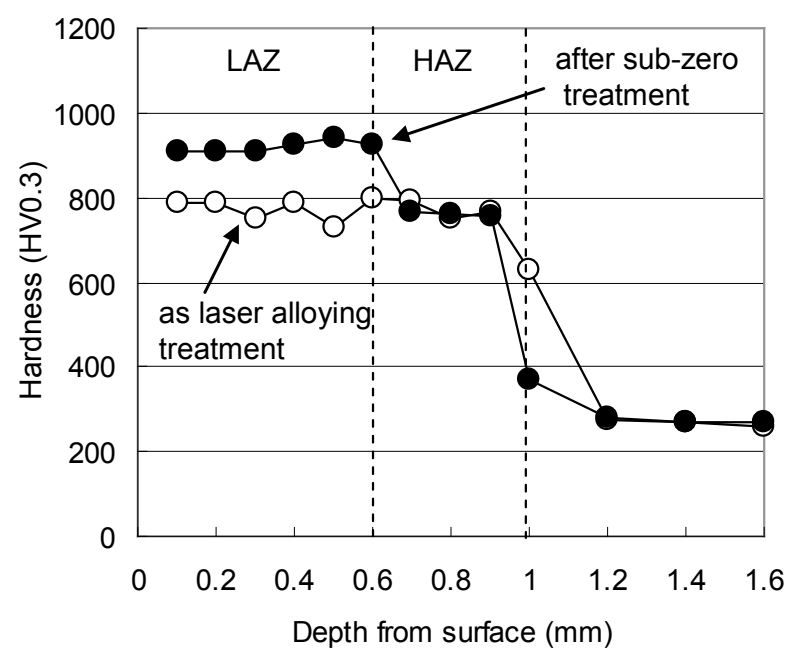

Fig.7 Effect of sub-zero treatment on hardness profile of $\mathrm{V}$ alloyed sample. (Traverse speed $5 \mathrm{~mm} / \mathrm{s}$ ) (LAZ:laser alloyed zone, HAZ:heat affected zone)

Fig.5 (d) and (f), fine lamellar structures of carbide and matrix were clearly observed. It seems that these structures were formed by eutectic solidification.

The lath martensite structure was observed in Tialloyed and $\mathrm{Nb}$-alloyed zone. This is the typical microstructure of medium carbon steel quenched from an austenite phase. On the other hand, in the matrix of V-alloyed zone, lath martensite structure was not observed.

From the analysis of the electron diffraction pattern, the matrix of the V-alloyed zone was a mainly fcc structure. It seems that, the matrix of the V-alloyed zone contains a lot of retained austenite phase due to decreasing of the Ms temperature by $\mathrm{V}$ or $\mathrm{C}$ enrichment.

\subsection{Hardness and wear property}

Fig. 6 and Fig. 7 show the hardness depth profiles of cross section from the samples. The hardness of both the laser alloyed zone and heat affected zone was higher than for the substrate. The high hardness of heat affected zone
Table 3 Conditions of wear testing.

\begin{tabular}{lc}
\hline Ball material & $\mathrm{Al}_{2} \mathrm{O}_{3}$ \\
Load & $9.8 \mathrm{~N}$ \\
Sliding speed & $2400 \mathrm{~mm} / \mathrm{min}$ \\
Amplitude & $5 \mathrm{~mm}$ \\
Sliding distance & $100 \mathrm{~m}$ \\
Lubricant & $\mathrm{dry}$ \\
Temperature & $25^{\circ} \mathrm{C}$ \\
Humidity & $50 \%$ \\
\hline
\end{tabular}

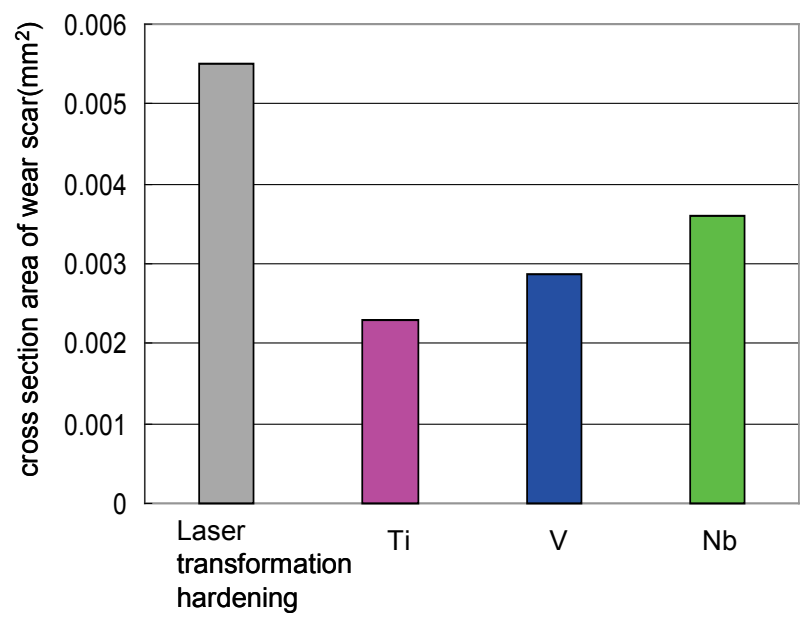

Fig.8 Comparison of wear amount.

was due to martensite transformation of the substrate caused by rapid cooling. The hardness of Ti-alloyed zone and $\mathrm{Nb}$-alloyed zone was much higher than that of the heat affected zone because of dispersion of hard MC carbide particles in the lath martensite matrix. The hardness of $\mathrm{V}$ alloyed zone was close to that of the heat affected zone, but the value was lower than that of $\mathrm{Ti}$ and $\mathrm{Nb}$ alloyed zone. However, after sub-zero treatment $\left(0.5\right.$ hour in liquid $\left.\mathrm{N}_{2}\right)$, the hardness of $\mathrm{V}$-alloyed zone increased over $900 \mathrm{HV}$ due to decomposition of retained austenite to martensite.

Wear testing using a reciprocating wear tester (ball-on flat) was carried out to evaluate the usefulness of the laser alloyed zone as a wear resistant material. Table 3 shows the conditions of wear testing. For comparison, a laser transformation hardening sample was prepared. The conditions for the laser transformation hardening were as follows: the laser beam diameter was $4.5 \mathrm{~mm}$, laser power was $720 \mathrm{~W}$, and the scanning speed was $8 \mathrm{~mm} / \mathrm{s}$.

The friction coefficient of the laser alloyed zone was about $0.50 \sim 0.65$, lower than that of laser transformation hardening sample 0.75. Fig. 8 shows the comparison of wear amount evaluated by the size of wear scar. The wear amount of laser alloyed zone was decreased compared with the laser transformation hardening sample. Carbide parti- 
cles are effective for decreasing the friction coefficient and improving wear resistance. The wear amount of each laser alloyed zone varies depending on the reinforcements.

\section{Conclusions}

Laser alloying using carbide forming elements (Ti, $\mathrm{Nb}$, and $\mathrm{V}$ ) was carried out to form an MC-Fe composite coating on a carbon steel substrate for improving wear resistance. The effect of additional elements on the carbide distribution and wear property of the laser alloyed zone was studied.

The laser alloyed zone contains MC carbide particles in an iron matrix. In Ti-alloyed and $\mathrm{Nb}$-alloyed zone, primary and eutectic carbides were observed in the lath martensite matrix. In V-alloyed zone, eutectic lamellar structure of carbide and iron were observed in the inter dendrite area of primary austenite. Hardness and wear properties of laser alloyed zone were improved as compared with the laser transformation hardened sample.

\section{Acknowledgements}

The authors would like to thank Dr. Yasuhiro Michiyama for wear testing experiments, and Dr. Yasunori Hasegawa for the STEM analysis.

\section{References}

[1] ASM Handbook Vol.18 (AMS International 1992) 801811.
[2] H. C. Man, S. Zhang, F. T. Cheng, T. M. Yue: Scr. Mater. 44 (2001) 2801-2807.

[3] L. Dubourg, D. Ursescu, F. Hlawka, A. Cornet: Wear 258 (2005) 1745-1754.

[4] H. Yan, A. Wang, Z. Xiong, K. Xu, Z. Huang: Appl. Surf. Sci. 256 (2010) 7001-7009.

[5] A. Emamian, S. F. Corbin, A. Khajepour: Surf. Coat. Technol. 206 (2012) 4495-4501.

[6] S. Nath, S. Pityana, J. D. Majumdar: Surf. Coat. Technol. 206 (2012) 3333-3341.

[7] C. W. Draper: Journal of Metals 34 (1982) 6 24-32.

[8] C. W. Draper, C. A. Ewing: J. Mater. Sci. 19 (1984) 3815-3825.

[9] W. M. Steen: Laser Material Processing third edition (Springer, 2003) 227-278.

[10] J. D. Ayers, T. R. Trucker: Thin Solid Films 73 (1980) 201-207.

[11] S. Ariely, J. Shen, M. Bamberger, F. Dausiger, H. Hugel: Surf. Coat. Technol. 45 (1991) 403-408.

[12] H. M. Flower, A. Walker, D. R. F. West: Scrip. Metall. 19 (1985) 923-926.

[13] V. M. Weerasinghe, D. R. F. West, J. de Damborenea: J. Mat. Proc. Technol. 58 (1996) 79-86.

[14] H. Holleck: J.Vac.Sci.Technol. A 4 (6) (1986) 26612669 .

[15] T. Yamaguchi, H. Hagino, M. Takemura, Y. Hasegawa, Y. Michiyama, A. Nakahira : Mater. Trans. 54 (2013) 1755-1759.

(Received: July 17, 2013, Accepted: March 10, 2014) 